\title{
Devoting attention to glucose variability and hypoglycaemia in type 2 diabetes
}

\author{
Martin K. Rutter ${ }^{1,2}$
}

Received: 8 August 2017 / Accepted: 23 August 2017 / Published online: 15 September 2017

(C) Springer-Verlag GmbH Germany 2017

\begin{abstract}
In the Trial Comparing Cardiovascular Safety of Insulin Degludec vs Insulin Glargine in Patients with Type 2 Diabetes at High Risk of Cardiovascular Events (DEVOTE), insulin degludec was non-inferior to insulin glargine in terms of cardiovascular events and mortality. However, there were lower rates of severe hypoglycaemia with insulin degludec. DEVOTE investigators now extend these findings by presenting the results of two observational epidemiological analyses based on trial data. In the first of these analyses (DEVOTE 2), Zinman et al (Diabetologia DOI: 10.1007/s00125-017-4423-z) demonstrate that, compared with individuals with lower dayto-day fasting glycaemic variability, those with higher day-today fasting glycaemic variability had a similar risk of major adverse cardiovascular events (MACE) but a higher risk of severe hypoglycaemia and all-cause mortality. In the second analysis (DEVOTE 3), Pieber et al (Diabetologia DOI: $10.1007 / \mathrm{s} 00125-017-4422-0$ ) found that individuals who experienced severe hypoglycaemia had a similar risk of MACE compared with those who never experienced severe hypoglycaemia, but had a more than twofold higher risk of subsequent total mortality and cardiovascular disease (CVD) mortality. The strengths of these studies relate to the availability of high-quality prospective data on adjudicated severe hypoglycaemia, MACE and mortality events in a large number of high-risk insulin-treated individuals with type 2 diabetes.
\end{abstract}

Martin K. Rutter

Martin.rutter@manchester.ac.uk

1 Division of Diabetes, Endocrinology and Gastroenterology, School of Medical Sciences, Faculty of Biology, Medicine and Health, University of Manchester, Manchester, UK

2 Manchester Diabetes Centre, 193 Hathersage Road, Central Manchester University hospitals NHS Foundation Trust, Manchester Academic Health Science Centre, Manchester M13 0JE, UK
Limitations include the observational nature of the data and thus residual confounding remains possible. Furthermore, the short duration of the trial resulted in limited statistical power for some analyses. Therefore, whilst DEVOTE 2 and DEVOTE 3 raise awareness of the mortality risks associated with glucose variability and severe hypoglycaemia in high-risk, insulintreated patients with type 2 diabetes, they cannot clarify causal relationships. Preventing severe hypoglycaemia in those with type 2 diabetes should already be a priority in clinical practice. However, findings from future clinical trials are needed to guide physicians on whether it is beneficial to target glucose variability, and risk for severe hypoglycaemia, to reduce the risks for CVD events and mortality in these individuals.

Keywords Cardiac complications · Cardiovascular risk · Clinical $\cdot$ Epidemiology $\cdot$ Glucose variability .

Hypoglycaemia $\cdot$ Insulin therapy $\cdot$ Mortality

\author{
Abbreviations \\ CVD Cardiovascular disease \\ DEVOTE Trial Comparing Cardiovascular Safety of \\ Insulin Degludec vs Insulin Glargine in Patients \\ with Type 2 Diabetes at High Risk of \\ Cardiovascular Events \\ MACE Major adverse cardiovascular event
}

\section{Background}

Individuals with type 2 diabetes continue to have a twofold higher risk of cardiovascular death and all-cause mortality compared with people without diabetes [1]. Attempts to reduce cardiovascular risk and mortality in type 2 diabetes through intensive glucose lowering have been disappointing, with trials 
suggesting only modest benefits [2]. In the case of the Action to Control Cardiovascular Risk in Diabetes (ACCORD) trial, intensive therapy was associated with higher mortality, which may have, in part, been owing to hypoglycaemia [3, 4].

Insulin is the most potent glucose-lowering therapy but its clinical use is limited by associated hypoglycaemia. Newer ultra-long-acting insulin therapies, with improved pharmacodynamic profiles, promise less glucose variability and lower risks of hypoglycaemia. Therefore, there is great interest in knowing whether these new insulin therapies provide cardiovascular and mortality benefits beyond those achieved with more traditional insulin therapies.

Against this backdrop, the results of the Trial Comparing Cardiovascular Safety of Insulin Degludec vs Insulin Glargine in Patients with Type 2 Diabetes at High Risk of Cardiovascular Events (DEVOTE) were recently reported [5]. This 2 year cardiovascular disease (CVD) safety trial found that insulin degludec was non-inferior to insulin glargine in terms of CVD events and mortality. However, although equivalent glycaemic control was achieved in the two groups, there was a $40 \%$ lower rate of severe hypoglycaemia and a 53\% lower rate of nocturnal severe hypoglycaemia with insulin degludec.

In this issue of Diabetologia, the DEVOTE investigators extend these findings by presenting the results of two observational epidemiological analyses based on DEVOTE trial data $[6,7]$. In addition to the importance of the research questions posed, major strengths of DEVOTE 2 and DEVOTE 3 relate to the availability of high-quality prospective data on adjudicated severe hypoglycaemia, major adverse cardiovascular events (MACE) and mortality events in a large number of high-risk insulin-treated individuals with type 2 diabetes. Both studies combined data on patients randomised to insulin degludec or insulin glargine after assessing that the trial allocation of these therapies did not influence the results.

\section{DEVOTE 2}

In DEVOTE 2 [6], the authors divided individuals into groups depending on whether they were classified as having high, medium or low glucose variability based on the result of three pre-breakfast capillary blood glucose values (recorded by participants each month). The risk of MACE, severe hypoglycaemia and all-cause mortality was assessed in relation to the standard deviation of fasting glucose variability. The authors found that higher day-to-day fasting glycaemic variability was associated with a higher risk of severe hypoglycaemia and all-cause mortality after adjusting for baseline differences in CVD risk factors, including $\mathrm{HbA}_{1 \mathrm{c}}$. However, higher glycaemic variability was not associated with MACE after adjusting for baseline and/or time varying risk factors, such as $\mathrm{HbA}_{1 \mathrm{c}}$.
DEVOTE 2 is the largest study to assess relationships between glucose variability and MACE/mortality in communityliving individuals with type 2 diabetes, and it is the largest study to relate fasting blood glucose variability with risk for severe hypoglycaemia [8-11]. However, it has some limitations: first, this was a post hoc analysis of trial data obtained for another purpose. This is important because there were many differences in baseline risk factors across high, medium and low glucose variability groups that could have influenced the study outcomes. Adjusting for some of these differences led to quite large attenuations in risk estimates suggesting a large influence of measured confounders. Residual confounding because of unmeasured variables could explain at least some of the observed relationships. Therefore, high glucose variability might simply be a marker of other conditions or behaviours leading to severe hypoglycaemia and higher mortality, such as autonomic neuropathy, cognitive dysfunction or frailty (Fig. 1). Consequently, these data cannot be interpreted as proof that glucose variability causes severe hypoglycaemia or higher mortality. Second, the data comes from a 2 year duration CVD safety trial designed to assess equivalence between two insulin therapies and, therefore, the statistical power to identify relationships between glucose variability and MACE/CVD mortality was limited. Third, as acknowledged by the authors, glucose variability was assessed from only three fasting blood glucose results each month; continuous glucose monitoring would have captured more data on glucose variability over $24 \mathrm{~h}$, including changes related to food intake, physical activity and therapy. Fourth, other studies have convincingly shown that glucose variability predicts severe hypoglycaemia and, therefore, these findings have less novelty than other findings.

Regarding mechanistic insights, there is a wealth of data from other sources relating glucose variability to oxidative stress, inflammation and thrombosis risk. These biological data potentially increase the likelihood that there is a genuine causal relationship between glucose variability and CVD risk/mortality but one must be cautious in such an interpretation as biological plausibility can be 'creatively' argued for many such questions. The data presented in DEVOTE 2 clearly confirm that fasting glucose variability is related to severe hypoglycaemia. We know from elegant studies, performed by Heller's group and others, that hypoglycaemia has the potential to cause fatal cardiac arrhythmias in those with type 2 diabetes [12-14]. Therefore, arguments related to biological plausibility somewhat support a causal link between glucose variability and CVD events/mortality. However, as emphasised above, the observational data presented in DEVOTE 2 do not take us closer to confirming causality.

Will the study change clinical practice? In type 1 diabetes the association between glucose variability and severe hypoglycaemia is fairly well understood in secondary care settings [15-17]. However, in individuals with type 2 diabetes, who 
Fig. 1 In DEVOTE 2, high glucose variability was related to a higher risk of severe hypoglycaemia and higher mortality. Explanations for these findings could involve common causes (confounding factors, such as autonomic neuropathy) and/or direct causal relationships

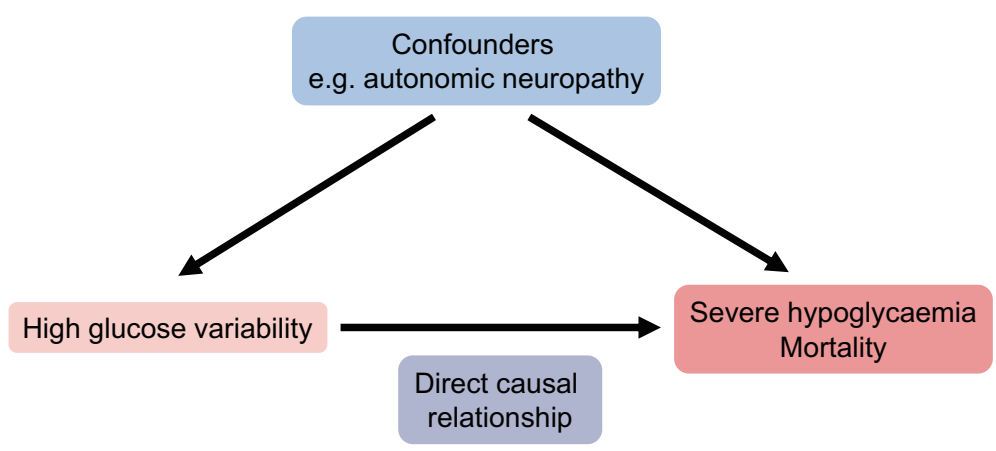

are mostly managed in primary care, this association is not 'on the radar' for most clinicians. The study should certainly make clinicians more aware of how glucose variability is associated with severe hypoglycaemia and mortality. However, in view of the uncertainty surrounding the nature of causal relationships, it would seem premature to recommend targeting glucose variability to reduce the risk of severe hypoglycaemia or mortality.

From a research perspective, a recent search on www. clinicaltrials.gov (search date 4 August 2017) indicates that several ongoing clinical trials in type 2 diabetes have a reduction in glucose variability as the primary endpoint, but none appear to target glucose variability to reduce CVD events. This lack of ongoing trials might reflect the practical issues of performing such trials, a lack of interest from pharmaceutical companies and/or the disappointing results from a previous trial targeting postprandial hyperglycaemia [18]. The Acarbose Cardiovascular Evaluation (ACE) trial is soon to report its results [19]. In this secondary prevention trial, 7500 people with impaired glucose tolerance and coronary artery disease were randomised to receive acarbose or placebo and were followed for MACE. Although the ACE trial also targets postprandial hyperglycaemia, a positive result could stimulate interest in trials targeting glucose variability to improve patient outcomes. However, one must recognise that acarbose may have other effects, such as lipid lowering, which could influence CVD outcomes. From a research perspective, DEVOTE 2 also highlights the need to standardise recording and reporting of glucose variability, as also suggested by others [20].

In summary, these new observational data from DEVOTE 2 have shown that fasting glucose variability was related to subsequent severe hypoglycaemia and mortality in people with type 2 diabetes. However, given the inherent limitations of observational data, only further trials can genuinely inform clinicians whether targeting glucose variability will reduce severe hypoglycaemia, CVD risk and mortality.

\section{DEVOTE 3}

In DEVOTE 3 [7], the temporal relationships between severe hypoglycaemia and MACE/all-cause mortality were evaluated.
During the study, 681 individuals experienced a MACE and 439 participants experienced $\geq 1$ episode of severe hypoglycaemia. Thirty-two patients had an episode of severe hypoglycaemia prior to a MACE, but only 16 patients experienced a MACE prior to an episode of severe hypoglycaemia. Of the 423 individuals who died, 38 did so after an episode of severe hypoglycaemia, sometimes many months after that episode.

When comparing people who experienced severe hypoglycaemia with those that did not, the relationship between severe hypoglycaemia and subsequent MACE was non-significant, even before adjusting for potential confounders. However, compared with individuals who never experienced severe hypoglycaemia during the study, those who experienced severe hypoglycaemia had a more than twofold higher risk of subsequent total mortality and CVD mortality. The relationship between severe hypoglycaemia and allcause mortality remained significant after adjusting for differences in several baseline risk factors between individuals with and without severe hypoglycaemia. The investigators did not analyse the data linking MACE to subsequent episodes of severe hypoglycaemia.

In addition to the strengths of this study mentioned above, DEVOTE 3 is novel in that it assessed the temporal relationships between severe hypoglycaemia and MACE/mortality [21]. However, the study also has several limitations, some of which are similar to those of DEVOTE 2: first, as with DEVOTE 2, this was a post hoc analysis of observational trial data. Differences in baseline risk factors between people with and without severe hypoglycaemia could explain relationships with MACE/mortality. Although the authors adjusted for several of these risk factors, residual confounding is a real possibility (Fig. 2). As acknowledged by the authors, causality cannot be inferred from this study: we do not know if those who died after severe hypoglycaemia died because of severe hypoglycaemia. Second, the analysis may have underestimated the risks associated with hypoglycaemia for three reasons: (1) the trial was of short duration and the number of MACE events that were linked to severe hypoglycaemia events was small and, thus, the power to detect relationships between severe hypoglycaemia and MACE events was low; (2) the study assessed the contribution of severe hypoglycaemia to outcomes 


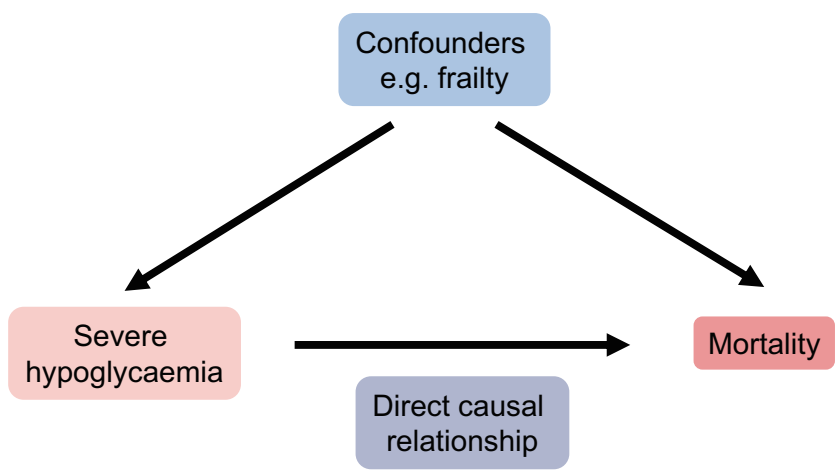

Fig. 2 In DEVOTE 3, severe hypoglycaemia was related to a higher risk of mortality. Explanations for this finding could involve common causes (confounding factors, such as frailty) and/or a direct causal relationship

but did not assess the contribution of non-severe hypo glycaemia episodes, which could have potentially led to prolonged QT intervals, repolarisation defects and arrhythmias [14]; (3) an analysis was not carried out of the individuals at highest-risk, who had multiple episodes of severe hypoglycaemia $(n=130$ experienced $\geq 2$ episodes of severe hypoglycaemia) [5]. Third, there was no explanation of how physicians managed episodes of severe hypoglycaemia to prevent further events - was this in line with standard clinical practice? [5]; fourth, the authors did not report the strengths of the associations between severe hypoglycaemia and outcomes after adjusting for potential confounders; and finally, although the numbers of events were small, it may have been appropriate to perform an exploratory analysis of the MACE events that were followed by severe hypoglycaemia. This would have created a more balanced analysis plan that was less focused on the potential causal pathway from severe hypoglycaemia to adverse outcomes.

Regarding mechanistic insights, the authors rightly point out the important physiological changes resulting from severe hypoglycaemia episodes that could lead to cardiac events and mortality, including autonomic, adrenal, prothrombotic and proinflammatory changes. However, these responses occur over hours or days, rather than over many months. Therefore, clinical intuition suggests that it is implausible that a single episode of severe hypoglycaemia could have an effect on mortality up to 1 year after the event. What seems more likely is that episodes of severe hypoglycaemia in this study are acting as a marker of frequent recurrent, but less severe hypoglycaemic episodes that may lead directly and rapidly to death. Alternatively, as indicated in Fig. 2 above, the severe hypoglycaemia episode might simply be a marker of other conditions or behaviours that lead to higher mortality risks, as suggested by others [22]. In their analysis, the authors adjusted for a small number of these potential confounders, such as renal impairment, but there was no adjustment for several potentially important factors, including autonomic neuropathy, cognitive function or frailty (as in DEVOTE 2).

Should the study influence clinical practice? Annual rates of severe hypoglycaemia in this high-risk trial population were in the range of what has been observed in clinical practice using different methods (5\% in DEVOTE vs $1-12 \%$ in clinical practice) [23-28]. Most physicians would agree that these rates are far too high. The main value of DEVOTE 3 is that it raises awareness of the potential mortality risks associated with severe hypoglycaemia in high-risk insulintreated patients with type 2 diabetes. Preventing severe hypoglycaemia in those with type 2 diabetes should already be a priority in clinical practice. However, detection and prevention of hypoglycaemia in type 2 diabetes is not given the attention that it deserves. Greater efforts should be made to identify individuals at high risk of hypoglycaemia, such as elderly individuals with cognitive impairment or renal disease. These patients could be harmed by use of complex therapies, including insulin [29, 30]. There is a need for better ways of detecting hypoglycaemia and, when appropriate, greater use of therapies that are associated with low risk of hypoglycaemia, including sodium-glucose cotransporter 2 (SGLT2) inhibitors, dipeptidyl peptidase-4 (DPP4) inhibitors, glucagon-like peptide 1 (GLP-1) agonists, and fast-acting and ultra-long-acting insulin analogues.

Regarding research implications, large cohort studies involving simultaneous continuous glucose monitoring and ECG monitoring could provide important insights into causal relationships between severe hypoglycaemia and cardiac outcomes, including arrhythmia, myocardial infarction and cardiac death. Clinical trials targeting risk for severe hypoglycaemia could quantify the benefits of clinical intervention on hypoglycaemia risk to reduce the risk for CVD events and mortality.

In summary, DEVOTE 3 raises awareness of the temporal association between severe hypoglycaemia and mortality in a large cohort of well characterised high-risk insulin-treated patients with type 2 diabetes.

\section{Conclusion}

Taken together, these observational studies raise awareness of the mortality risks associated with glucose variability and severe hypoglycaemia in high-risk insulin-treated patients with type 2 diabetes but they do not clarify causal relationships. Only the results of further clinical trials can genuinely guide physicians on whether to target glucose variability and risk for severe hypoglycaemia to reduce the risks for CVD events and mortality in these individuals. 
Acknowledgements The author would like to thank N. Sattar (University of Oxford and Oxford University Hospitals NHS Foundation Trust), L. Leelarathna (Central Manchester University Hospitals NHS Foundation Trust [CMFT], University of Manchester and Manchester Academic Health Science Centre [MAHSC]); and H. Thabit (CMFT, University of Manchester and MAHSC) for their helpful suggestions in the preparation of this manuscript.

Funding The author receives financial support from the University of Manchester and CMFT.

Duality of interest The author reports receiving honoraria and funding to attend educational meetings from Novo Nordisk, and honoraria and consulting fees from Ascensia, Cell Catapult and Roche Diabetes Care.

Contribution statement The author was the sole contributor to this paper.

\section{References}

1. Wright AK, Kontopantelis E, Emsley R et al (2017) Life expectancy and cause-specific mortality in type 2 diabetes: a populationbased cohort study quantifying relationships in ethnic subgroups. Diabetes Care 40:338-345

2. Hemmingsen B, Lund SS, Gluud C et al (2011) Intensive glycaemic control for patients with type 2 diabetes: systematic review with meta-analysis and trial sequential analysis of randomised clinical trials. BMJ d6898:343

3. Gerstein HC, Miller ME, Byington RP et al (2008) Effects of intensive glucose lowering in type 2 diabetes. N Engl J Med 358: 2545-2559

4. Bonds DE, Miller ME, Bergenstal RM et al (2010) The association between symptomatic, severe hypoglycaemia and mortality in type 2 diabetes: retrospective epidemiological analysis of the ACCORD study. BMJ b4909:340

5. Marso SP, McGuire DK, Zinman B et al (2017) Efficacy and safety of degludec versus glargine in type 2 diabetes. N Engl J Med 377: 723-732

6. Zinman B, Marso SP, Poulter NR, et al (2017) Day-to-day fasting glycaemic variability in DEVOTE: associations with severe hypoglycaemia and cardiovascular outcomes (DEVOTE 2). Diabetologia. doi:10.1007/s00125-017-4423-Z

7. Pieber TR, Marso SP, McGuire DK, et al (2017) DEVOTE 3: temporal relationships between severe hypoglycaemia, cardiovascular outcomes and mortality. Diabetologia. doi:10.1007/s00125-017$4422-0$

8. Xu D, Fang H, Xu W, Yan Y, Liu Y, Yao B (2016) Fasting plasma glucose variability and all-cause mortality among type 2 diabetes patients: a dynamic cohort study in Shanghai, China. Sci Rep 6: 39633

9. Tschope D, Bramlage P, Schneider S, Gitt AK (2016) Incidence, characteristics and impact of hypoglycaemia in patients receiving intensified treatment for inadequately controlled type 2 diabetes mellitus. Diab Vasc Dis Res 13:2-12

10. Siegelaar SE, Holleman F, Hoekstra JB, DeVries JH (2010) Glucose variability; does it matter? Endocr Rev 31:171-182

11. Nalysnyk L, Hernandez-Medina M, Krishnarajah G (2010) Glycaemic variability and complications in patients with diabetes mellitus: evidence from a systematic review of the literature. Diabetes Obes Metab 12:288-298
12. Stahn A, Pistrosch F, Ganz X et al (2014) Relationship between hypoglycemic episodes and ventricular arrhythmias in patients with type 2 diabetes and cardiovascular diseases: silent hypoglycemias and silent arrhythmias. Diabetes Care 37:516-520

13. Chow E, Bernjak A, Walkinshaw E et al (2017) Cardiac autonomic regulation and repolarization during acute experimental hypoglycemia in type 2 diabetes. Diabetes 66:1322-1333

14. Chow E, Bernjak A, Williams S et al (2014) Risk of cardiac arrhythmias during hypoglycemia in patients with type 2 diabetes and cardiovascular risk. Diabetes 63:1738-1747

15. Weinstock RS, DuBose SN, Bergenstal RM et al (2016) Risk factors associated with severe hypoglycemia in older adults with type 1 diabetes. Diabetes Care 39:603-610

16. DuBose SN, Weinstock RS, Beck RW et al (2016) Hypoglycemia in older adults with type 1 diabetes. Diabetes Technol Ther 18:765771

17. Suh S, Kim JH (2015) Glycemic variability: how do we measure it and why is it important? Diabetes Metab J 39:273-282

18. Raz I, Wilson PW, Strojek K et al (2009) Effects of prandial versus fasting glycemia on cardiovascular outcomes in type 2 diabetes: the HEART2D trial. Diabetes Care 32:381-386

19. Holman RR, Bethel MA, Chan JC et al (2014) Rationale for and design of the Acarbose Cardiovascular Evaluation (ACE) trial. Am Heart J 168:23-29 e22

20. Schnell O, Barnard K, Bergenstal R et al (2017) Role of continuous glucose monitoring in clinical trials: recommendations on reporting. Diabetes Technol Ther 19:391-399

21. Goto A, Arah OA, Goto M, Terauchi Y, Noda M (2013) Severe hypoglycaemia and cardiovascular disease: systematic review and meta-analysis with bias analysis. BMJ 347:f4533

22. Zoungas S, Patel A, Chalmers J et al (2010) Severe hypoglycemia and risks of vascular events and death. N Engl J Med 363:1410 1418

23. Karter AJ, Lipska KJ, O'Connor PJ et al (2017) High rates of severe hypoglycemia among African American patients with diabetes: the surveillance, prevention, and Management of Diabetes Mellitus (SUPREME-DM) network. J Diabetes Complicat 31:869-873

24. Pathak RD, Schroeder EB, Seaquist ER et al (2016) Severe hypoglycemia requiring medical intervention in a large cohort of adults with diabetes receiving care in U.S. integrated health care delivery systems: 2005-2011. Diabetes Care 39:363-370

25. Simon D, de Pablos-Velasco P, Parhofer KG et al (2015) Hypoglycaemic episodes in patients with type 2 diabetes - risk factors and associations with patient-reported outcomes: the PANORAMA study. Diabete Metab 41:470-479

26. Donnelly LA, Morris AD, Frier BM et al (2005) Frequency and predictors of hypoglycaemia in type 1 and insulin-treated type 2 diabetes: a population-based study. Diabet Med 22:749-755

27. Leese GP, Wang J, Broomhall J et al (2003) Frequency of severe hypoglycemia requiring emergency treatment in type 1 and type 2 diabetes: a population-based study of health service resource use. Diabetes Care 26:1176-1180

28. UK Hypoglycaemia Study Group (2007) Risk of hypoglycaemia in types 1 and 2 diabetes: effects of treatment modalities and their duration. Diabetologia 50:1140-1147

29. McCoy RG, Lipska KJ, Yao X, Ross JS, Montori VM, Shah ND (2016) Intensive treatment and severe hypoglycemia among adults with type 2 diabetes. JAMA Intern Med 176:969-978

30. Hippisley-Cox J, Coupland C (2016) Diabetes treatments and risk of amputation, blindness, severe kidney failure, hyperglycaemia, and hypoglycaemia: open cohort study in primary care. BMJ 352 : i1450 